S. BRUNiAUX, D. LUART, C. LEN* (UNIVERSité DE TECHNOLOGIE DE COMPIÈGNe, ECOLE SUPÉRIEURE DE CHIMIE ORGANIQUE ET MINÉRALE, COMPIÈGNE, AND PSL RESEARCH UNIVERSITY, PARIS, FRANCE)

Continuous-Flow Reductive Alkylation: Synthesis of Bio-Based Symmetrical and Dissymmetrical Ethers Synthesis 2018, 50, 1849-1856.

\section{Continuous-Flow Pd/C-Catalyzed Reductive Alkylation of Alcohols with Aldehydes}

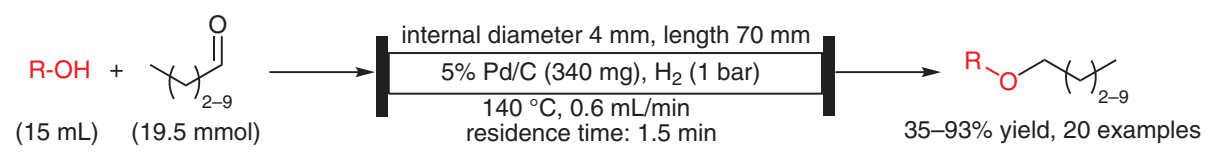
Results:

$$
\widehat{\mathrm{O}} \widehat{\mathrm{HY}_{2}}
$$

$79 \%$ yield

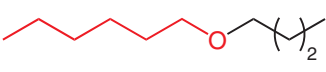

$67 \%$ yield<smiles>CCCOCCN</smiles>

$88 \%$ yield<smiles>CCCCOCC(CC)CCCC</smiles>

$46 \%$ yield<smiles>CCCCC(C)OCCC</smiles>

$43 \%$ yield

$$
\widehat{\mathrm{O}} \mathrm{NH}_{4}
$$

$68 \%$ yield

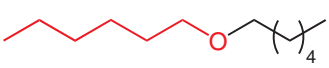

$58 \%$ yield<smiles>CCCOCC(C)C</smiles>

$59 \%$ yield<smiles>CCCCOCC(CC)CCCC</smiles>

$35 \%$ yield<smiles>CCCCC(C)OCC(C)C</smiles>

$43 \%$ yield<smiles>CCCCOCC(C)C</smiles>

$52 \%$ yield

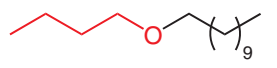
$43 \%$ yield<smiles>CCCCCCOCCCC</smiles>

$50 \%$ yield

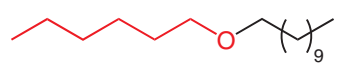<smiles>CCCCCCCCCCCOCCC(C)C</smiles>

$71 \%$ yield<smiles>CC(C)CCOCC(C)C</smiles>

$57 \%$ yield<smiles>CC(C)COC(C)C</smiles>

$57 \%$ yield<smiles>CCCCOC(C)CC</smiles>

$70 \%$ yield

$55 \%$ yield
Significance: The authors have developed a continuous-flow system for the reductive alkylation of alcohols with aldehydes catalyzed by $\mathrm{Pd} / \mathrm{C}$ in the presence of hydrogen, giving the corresponding ethers in 35-93\% yield.
Gategory

Polymer-Supported Synthesis

\section{Key words}

palladium catalysis

flow reaction

reductive alkylation

ethers

green chemistry 